PROCEEDINGS OF THE

AMERICAN MATHEMATICAL SOCIETY

Volume 125, Number 4, April 1997, Pages 1167-1176

S $0002-9939(97) 03696-4$

\title{
ON THE NON-EXISTENCE OF HOMOCLINIC ORBITS FOR A CLASS OF INFINITE DIMENSIONAL HAMILTONIAN SYSTEMS
}

\author{
PH. CLÉMENT AND R.C.A.M. VAN DER VORST
}

(Communicated by Hal L. Smith)

\begin{abstract}
We prove that for a class of infinite dimensional Hamiltonian systems certain homoclinic connections to the origin cease to exist when the nonlinearities have 'super-critical' growth. The proof is based on a variational principle and a Pohožaev type identity.
\end{abstract}

\section{INTRODUCTION}

In a recent paper Clément, Felmer and Mitidieri [2] studied the existence of homoclinic orbits of the following infinite dimensional Hamiltonian system:

$$
(\mathrm{H}) \begin{cases}-\frac{\partial v}{\partial t}-\Delta v=|u|^{p-1} u, & \text { in } \Omega \times \mathbf{R}, \\ \frac{\partial u}{\partial t}-\Delta u=|v|^{q-1} v, & \text { in } \Omega \times \mathbf{R}, \\ u(x, t)=v(x, t)=0, & \text { on } \partial \Omega \times \mathbf{R}, \\ \lim _{t \rightarrow \pm \infty} u(t, x)=\lim _{t \rightarrow \pm \infty} v(t, x)=0, & \text { uniformly in } x \in \Omega,\end{cases}
$$

where $\Omega \subset \mathbf{R}^{n}, n \geq 1$, is a bounded domain with smooth boundary $\partial \Omega$ and $p q>1$, $p, q>0$. Homoclinic connections to the origin are solutions $u, v \in C^{2,1}(\bar{\Omega} \times \mathbf{R})$. These solutions will also be referred to as classical solutions of System $(\mathrm{H})$. The main result in [2] is:

Theorem 1.1. Assume that $p, q$ satisfy

$$
1>\frac{1}{p+1}+\frac{1}{q+1}>\frac{n}{n+2}, \quad p, q>0 .
$$

Then System (H) has at least one non-trivial classical solution (homoclinic connection to the origin) with positive components.

In this note we shall prove that (1.5) is indeed 'optimal' for the existence of homoclinic connections to the origin. When (1.5) does not hold and $\Omega$ is starshaped, System $(\mathrm{H})$ does not have classical solutions with positive components. Our main result can be stated as follows:

Received by the editors October 25, 1995.

1991 Mathematics Subject Classification. Primary 35J50, 35J55, 46E35.

This work was supported by the Netherlands Organization for Scientific Research, NWO and EC-HCM project Reaction-Diffusion Equations ERBCHRXCT930409.

(C)1997 American Mathematical Society 
Theorem 1.2. Let $\Omega \subset \mathbf{R}^{n}$ be star-shaped and assume that $p, q$ satisfy

$$
\frac{1}{p+1}+\frac{1}{q+1} \leq \frac{n}{n+2}, \quad p, q>0 .
$$

Then System (H) has no homoclinic connections to the origin with positive components.

The relations (1.5) and (1.6) are complementary, which proves the sharpness of both Theorem 1.1 and 1.2 in the case when $\Omega$ is star-shaped. The case of nonstarshaped domains is much more delicate and will not be considered here (e.g., see [1] for elliptic equations).

Observe that the hyperbola of critical exponents

$$
\frac{1}{p+1}+\frac{1}{q+1}=\frac{n}{n+2}, \quad p, q>0,
$$

is different from the one for the stationary equations (e.g., see [5], [12]), since our domain is $\Omega \times \mathbf{R}$ instead of $\Omega$. If one considers the case $q=1$ one can rewrite the equations (1.1) and (1.2) into a hypo-elliptic non-homogeneous 2nd/4th order equation in the strip $\Omega \times \mathbf{R}$. For this equation the theory of Pucci-Serrin [9] can be applied and gives the same critical exponent as relation (1.7) for $q=1$.

The equations (1.1) and (1.2) can be derived from a variational principle, i.e. they can be viewed as the Euler-Lagrange equations of the functional

$$
J(u, v)=\int_{\mathbf{R}} \int_{\Omega}\left(v \frac{\partial u}{\partial t}+\nabla u \nabla v-\frac{1}{p+1}|u|^{p+1}-\frac{1}{q+1}|v|^{q+1}\right) d x d t,
$$

where the integrand will be denoted by $\mathcal{J}$. The integrand $\mathcal{J}$ is called the Lagrangian density of System (H). Observe that if $\Omega=\mathbf{R}^{n}$ and the $p, q$ satisfy (1.7), then the functional $J$ is invariant under the transformations

$$
u(x, t) \longrightarrow e^{-\frac{n+2}{p+1} \varepsilon} u\left(e^{-\varepsilon} x, e^{-2 \varepsilon} t\right), \quad v(x, t) \longrightarrow e^{-\frac{n+2}{q+1} \varepsilon} v\left(e^{-\varepsilon} x, e^{-2 \varepsilon} t\right),
$$

with $\varepsilon \in(\mathbf{R},+)$. In this situation the celebrated Noether's Theorem [6] provides a conservation law for System $(\mathrm{H})$. However our system is not invariant under the transformations given by (1.9). The adaptation of Noether's Theorem as described in [4] and [12] (see also [5] and [9]) will be used to obtain an almost conservation law, which can be interpreted as a Pohožaev type identity (see [8], [12]).

Since System $(\mathrm{H})$ is autonomous, the functional $J$ is invariant under time-translations, i.e. the transformations

$$
u(x, t) \longrightarrow u(x, t+\tau), \quad v(x, t) \longrightarrow v(x, t+\tau), \quad \tau \in \mathbf{R},
$$

leave $J$ invariant. Again from Noether's Theorem one obtains a conservation law, given by

$$
H(u, v)=\int_{\Omega}\left(\nabla u \nabla v-\frac{1}{p+1}|u|^{p+1}-\frac{1}{q+1}|v|^{q+1}\right) d x=\text { constant },
$$

which shall be referred to as the Hamiltonian of System $(\mathrm{H})$.

If one replaces the boundary conditions (1.3) by $u(x, t)=\frac{\partial u}{\partial n}(x, t)=0$ on $\partial \Omega \times \mathbf{R}$, one can prove that System $(\mathrm{H})$ does not have homoclinic connections when $\frac{1}{p+1}+$ $\frac{1}{q+1}<\frac{n}{n+2}$ and $\Omega$ is star-shaped. In order to handle the critical case one needs a unique continuation result for the linear differential operator in System $(\mathrm{H})$. 


\section{Almost invariance And Noether's Theorem}

As was mentioned in Section 1, the transformations (1.9) only leave $J$ invariant when $p, q$ satisfy (1.7). Invariance means that the 'Lie-derivative' of $J$ with respect to this transformation group is zero at (classical) solutions of System $(\mathrm{H})$. In the case that (1.7) is not satisfied one can still compute the Lie-derivative of $J$ with respect to the transformation group given by (1.9).

In order to do so we shall now use the Lagrangian density $\mathcal{J}\left(u, v, \nabla u, \nabla v, u_{t}, v_{t}\right)$. The variables $u, v x^{i}$ and $t$ will be considered as the independent variables, and $p_{u}^{i}=\nabla u, p_{v}^{i}=\nabla v, p_{u}^{t}=u_{t}$ and $p_{v}^{t}=v_{t}$ as the dependent variables. The transformations given by (1.9) are equivalent to the following set of transformations of the independent variables $u, v, x^{i}, t$ :

$$
\begin{array}{ll}
\tilde{u}=e^{-\alpha \varepsilon} u, & \tilde{x}^{i}=e^{\varepsilon} x^{i}, \\
\tilde{v}=e^{-\beta \varepsilon} v, & \tilde{t}=e^{2 \varepsilon} t,
\end{array}
$$

where $\alpha=\frac{n+2}{p^{*}+1}, \beta=\frac{n+2}{q^{*}+1}$ and $p^{*}, q^{*}$ satisfy (1.7). We write $g_{\varepsilon}\left(u, v, x^{i}, t\right)=$ $\left(\tilde{u}, \tilde{v}, \tilde{x}^{i}, \tilde{t}\right)$. The infinitesimal generator of $g_{\varepsilon}$ is given by (see e.g. [4], [7])

$$
\mathbf{v}=x^{i} \frac{\partial}{\partial x^{i}}+2 t \frac{\partial}{\partial t}-\alpha u \frac{\partial}{\partial u}-\beta v \frac{\partial}{\partial v} .
$$

The way the variables $p_{u}^{i}, p_{v}^{i}, p_{u}^{t}$ and $p_{v}^{t}$ transform now will depend on (2.1) and (2.2). The Lie-derivative of $\mathcal{J}$ with respect to $g_{\varepsilon}$ is defined by

$$
D_{g_{\varepsilon}} \mathcal{J}=\left.\frac{d}{d \varepsilon}\left[\mathcal{J}\left(g_{\varepsilon}\left(u, v, x^{i}, t\right)\right) \frac{d \tilde{x}}{d x} \frac{d \tilde{t}}{d t}\right]\right|_{\varepsilon=0},
$$

where $\frac{d \tilde{x}}{d x}=\operatorname{det}\left(\frac{\partial \tilde{x}^{i}}{\partial x^{i}}\right)$. If $p_{u}^{i}, p_{v}^{i}, p_{u}^{t}$ and $p_{v}^{t}$ were independent variables (which would not be transformed), one can compute the Lie-derivative of $\mathcal{J}$ at a classical solution by simply applying the differential operator $\mathbf{v}$, given by (2.3), to $\mathcal{J}$. However $\mathcal{J}$ depends also on the dependent variables $p_{u}^{i}, p_{v}^{i}, p_{u}^{t}$ and $p_{v}^{t}$, so that one needs to extend the infinitesimal generator $\mathbf{v}$ in order to cope with the dependent variables. The extension of $\mathbf{v}$ is denoted by $\mathbf{v}^{(1)}$ and is called the prolonged vectorfield (see [7] for an explicit formula). The expression for the Lie-derivative now becomes

$$
D_{g_{\varepsilon}} \mathcal{J}=\mathbf{v}^{(1)} \mathcal{J}+(n+2) \mathcal{J} .
$$

Applying the transformations (2.1) and (2.2) to $\mathcal{J}$ directly and computing $D_{g_{\varepsilon}} \mathcal{J}$ according to (2.4) obviously gives the same result. One may choose either of these two ways to compute $D_{g_{\varepsilon}} \mathcal{J}$.

Lemma 2.1. Let $u, v \in C^{2,1}(\bar{\Omega} \times \mathbf{R})$ be a classical solution of System $(H)$. Then

$$
D_{g_{\varepsilon}} \mathcal{J}=\left(\alpha-\frac{n+2}{p+1}\right)|u|^{p+1}+\left(\beta-\frac{n+2}{q+1}\right)|v|^{q+1}, \forall(x, t) \in \Omega \times \mathbf{R},
$$

with $\alpha+\beta=n$.

Proof of Lemma 2.1. In order to prove this lemma we can apply Theorem 2.1 of [12] using the infinitesimal generator $\mathbf{v}$. Instead we will compute $\mathcal{J}\left(g_{\varepsilon}\left(u, v, x^{i}, t\right)\right) \frac{d \tilde{x}}{d x} \frac{d \tilde{t}}{d t}$ 
and determine its derivative at $\varepsilon=0$. Using (2.1) and (2.2), we find that

$$
\begin{aligned}
\mathcal{J}\left(g_{\varepsilon}\left(u, v, x^{i}, t\right)\right) \frac{d \tilde{x}}{d x} \frac{d \tilde{t}}{d t} & =v \frac{\partial u}{\partial t}+\nabla u \nabla v \\
& -\frac{e^{(-(p+1) \alpha+n+2) \varepsilon}}{p+1}|u|^{p+1}-\frac{e^{(-(q+1) \beta+n+2) \varepsilon}}{q+1}|v|^{q+1},
\end{aligned}
$$

where $\frac{d \tilde{x}}{d x}=e^{n \varepsilon}$. Applying (2.4), we immediately obtain (2.6).

From Lemma 2.1 it immediately follows that the Lie-derivative $D_{g_{\varepsilon}} \mathcal{J}$ is positive (positive pointwise in $\Omega \times \mathbf{R}$ ) when $p, q$ satisfy (1.6) with strict inequality, and identically equal to zero when $p, q$ satisfy (1.7). Indeed if $p, q$ satisfy (1.6) with strict inequality, one can choose numbers $p^{*}$ and $q^{*}$, which satisfy (1.7), such that $\frac{1}{p+1}<\frac{1}{p^{*}+1}$ and $\frac{1}{q+1}<\frac{1}{q^{*}+1}$. Taking $\alpha=\frac{n+2}{p^{*}+1}$ and $\beta=\frac{n+2}{q^{*}+1}$ then gives the desired result. In the case that $p$ and $q$ satisfy (1.7) one chooses $\alpha=\frac{n+2}{p+1}$ and $\beta=\frac{n+2}{q+1}$.

If we now apply the modified version of Noether's Theorem (see [4], [12]), we obtain the local form of a Pohožaev type identity for solutions of System $(\mathrm{H})$.

Lemma 2.2. Let $u, v \in C^{2,1}(\bar{\Omega} \times \mathbf{R})$ be a classical solution of System (H) and let

$$
\mathbf{P}=\left(P^{i}, P^{t}\right)=\left(x^{i} \mathcal{J}+Q_{u} \frac{\partial \mathcal{J}}{\partial p_{u}^{i}}+Q_{v} \frac{\partial \mathcal{J}}{\partial p_{v}^{i}}, 2 t \mathcal{J}+Q_{u} \frac{\partial \mathcal{J}}{\partial p_{u}^{t}}+Q_{v} \frac{\partial \mathcal{J}}{\partial p_{v}^{t}}\right),
$$

with $Q_{u}=-\alpha u-p_{u}^{i} x^{i}-2 t p_{u}^{t}, Q_{v}=-\beta v-p_{v}^{i} x^{i}-2 t p_{v}^{t}$. Then,

$$
\operatorname{div} \mathbf{P}=D_{g_{\varepsilon}} \mathcal{J}=\left(\alpha-\frac{n+2}{p+1}\right)|u|^{p+1}+\left(\beta-\frac{n+2}{q+1}\right)|v|^{q+1}, \forall(x, t) \in \Omega \times \mathbf{R} .
$$

Proof of Lemma 2.2. See [4] and [12].

If one determines the expressions for $P^{i}$ and $P^{t}$, it follows that

$$
\begin{aligned}
P^{i}= & x^{i} v \frac{\partial u}{\partial t}+x^{i} \nabla u \nabla v-\frac{x^{i}}{p+1}|u|^{p+1}-\frac{x^{i}}{q+1}|v|^{q+1} \\
& -\left(x \cdot \nabla u+2 t \frac{\partial u}{\partial t}\right) \nabla v-\left(x \cdot \nabla v+2 t \frac{\partial v}{\partial t}\right) \nabla u-\alpha u \nabla v-\beta v \nabla u, \\
P^{t}= & 2 t v \frac{\partial u}{\partial t}+2 t \nabla u \nabla v-\frac{2 t}{p+1}|u|^{p+1}-\frac{2 t}{q+1}|v|^{q+1} \\
& -\left(x \cdot \nabla u+2 t \frac{\partial u}{\partial t}\right) v-\alpha u v .
\end{aligned}
$$

In the next section we shall use these expressions in order to obtain an integral identity for solutions of System (H).

\section{The integral IDentity and the Proof of Theorem 1.2}

In order to integrate (2.8) one needs certain a priori integrability properties for classical solutions of System (H). Since $\Omega$ is bounded and the solutions $u, v$ are continuous up to the boundary in $x, L^{r}$-integrability in $x$ for any $r \geq 1$ is easily obtained. However, since the domain of definition of the time-variable is $\mathbf{R}$, the integrability properties are not immediately clear. A precise analysis of the asymptotic behavior of a solution $(u, v)$ near the trivial solution $(0,0)$ will give us the required information. It follows, if $p q>1$ (or equivalently $\frac{1}{p+1}+\frac{1}{q+1}<1$ ), that $u$ and $v$ converge to zero as $t \rightarrow \pm \infty$, uniformly in $x$ with exponential decay in the $t$-variable. For the case $p, q \geq 1$ this is an obvious byproduct of the fact 

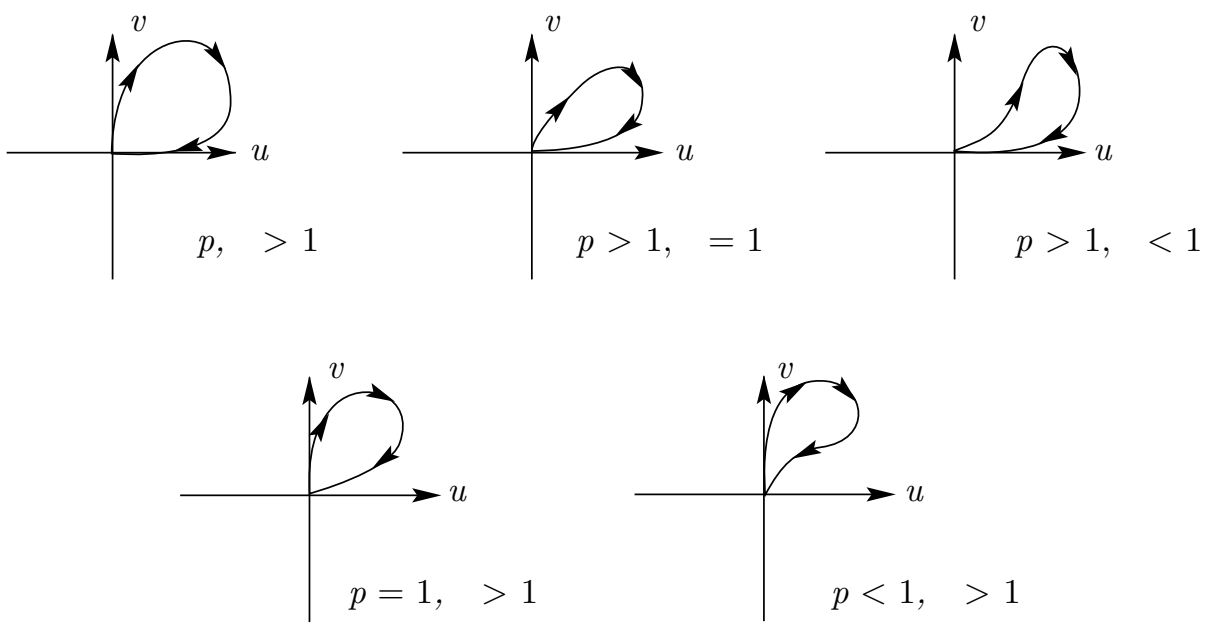

Figure 1

that $(u, v)(x, t)$ approach the origin along the local stable and unstable manifold of the origin as $t \rightarrow \pm \infty$ respectively (see [3]). However if $p<1(q>1)$ or if $q<1$ $(p>1)$, one cannot apply the above result, although the asymptotic behavior is still exponential in time (see Fig. 1).

Lemma 3.1. Let $u, v \in C^{2,1}(\bar{\Omega} \times \mathbf{R})$ be a classical solution of System (H). Then there exist $t_{0}>0$ and constants $C_{1}, \ldots, C_{4}>0$ such that

$$
\sup _{x \in \bar{\Omega}}|u(x, t)| \leq C_{1} e^{-\gamma\left(t-t_{0}\right)}, \quad \sup _{x \in \bar{\Omega}}|v(x, t)| \leq C_{2} e^{-\gamma p\left(t-t_{0}\right)}, t \geq t_{0},
$$

and

$$
\sup _{x \in \bar{\Omega}}|u(x, t)| \leq C_{3} e^{\gamma q\left(t+t_{0}\right)}, \quad \sup _{x \in \bar{\Omega}}|v(x, t)| \leq C_{4} e^{\gamma\left(t+t_{0}\right)},-t \geq t_{0},
$$

for any $0<\gamma<\lambda_{1}$, where $\lambda_{1}$ is the principal eigenvalue of $-\Delta$ with Dirichlet boundary conditions.

Proof of Lemma 3.1. For any $1<r<\infty$ the operator $-\Delta$ (with Dirichlet boundary conditions) is sectorial on $L^{r}(\Omega)[3]$ with $D(\Delta)=W^{2, r} \cap W_{0}^{1, r}(\Omega)=X_{1}$, and its spectrum $\sigma(-\Delta)$ is contained in the half-plane $\left\{\lambda ; \operatorname{Re}(\lambda)>\gamma^{\prime}, 0<\gamma^{\prime}<\lambda_{1}\right\}$, where $\lambda_{1}$ is the principal eigenvalue of $-\Delta$. The operator $e^{t \Delta}$ satisfies the estimate

$$
\left\|e^{t \Delta} u\right\|_{L^{r}} \leq M_{r} e^{-\gamma^{\prime} t}\|u\|_{L^{r}},
$$

with $0<\gamma^{\prime}<\lambda_{1}$ and $M_{r} \geq 1$. From the boundedness of $u(t)$ and $v(t)$ in $L^{r}(\Omega)$ and from the previous estimate on $e^{t \Delta}$ it follows that the solutions of System $(\mathrm{H})$ verify the integral equations

$$
\begin{aligned}
& u(t)=e^{\left(t-t_{0}\right) \Delta} u\left(t_{0}\right)+\int_{t_{0}}^{t} e^{(t-s) \Delta} v^{q}(s) d s, \\
& v(t)=\int_{t}^{\infty} e^{(s-t) \Delta} u^{p}(s) d s,
\end{aligned}
$$


for all $t \geq t_{0}$, where $t_{0}$ is arbitrary real number. We claim that there exist numbers $\gamma \in\left(0, \gamma^{\prime}\right), \rho>0$ and $t_{0} \in \mathbf{R}$ such that the following estimate holds:

$$
\|u(t)\|_{L^{r}} \leq 2 M_{r} e^{-\gamma\left(t-t_{0}\right)} \rho+2^{-n+1} M_{r} \rho, \quad \forall t \geq t_{0}, \quad \forall n \geq 1 .
$$

We shall prove (3.5) by induction. First we choose $\gamma \in\left(0, \gamma^{\prime}\right)$, and since $p q>1$, there exists a $\rho>0$ satisfying

$$
\left[5 M\left(\frac{M^{\prime}}{\gamma^{\prime}}\right)^{q} \frac{1}{\gamma^{\prime}-\gamma} \rho^{p q-1}\right]<1 / 2,
$$

where $M=M_{r}$ and $M^{\prime}=M_{r q}$. Finally we choose $t_{0}$ large enough so that $\|u(t)\|_{L^{r}} \leq \rho$, for $t \geq t_{0}$. With this of choice $t_{0}, \rho$ and $\gamma,(3.5)$ holds for $n=1$. From (3.3) and (3.4) it follows that

$$
\begin{aligned}
\|u(t)\|_{L^{r}} & \leq M e^{-\gamma^{\prime}\left(t-t_{0}\right)}\left\|u\left(t_{0}\right)\right\|_{L^{r}}+M \int_{t_{0}}^{t} e^{-\gamma^{\prime}(t-s)}\left\|v^{q}(s)\right\|_{L^{r}} d s \\
& \leq M e^{-\gamma\left(t-t_{0}\right)}\left\|u\left(t_{0}\right)\right\|_{L^{r}}+M \int_{t_{0}}^{t} e^{-\gamma^{\prime}(t-s)}\|v(s)\|_{L^{r q}}^{q} d s, \\
\|v(s)\|_{L^{r q}} & \leq M^{\prime} \int_{s}^{\infty} e^{-\gamma^{\prime}(\tau-s)}\left\|u^{p}(\tau)\right\|_{L^{r q}} d \tau \\
& =M^{\prime} \int_{s}^{\infty} e^{-\gamma^{\prime}(\tau-s)}\left\|u^{p q}(\tau)\right\|_{L^{r}}^{1 / q} d \tau .
\end{aligned}
$$

Notice, since $p q>1$ and $\|u(t)\|_{L^{r}} \leq \rho$, that $\left\|u^{p q}(t)\right\|_{L^{r}} \leq \rho^{p q-1}\|u(t)\|_{L^{r}}$ and therefore (3.7) becomes

$$
\|v(s)\|_{L^{r q}}^{q} \leq\left(\frac{M^{\prime}}{\gamma^{\prime}}\right)^{q} \rho^{p q-1} \sup _{[s, \infty)}\|u(\tau)\|_{L^{r}} .
$$

At this point we assume that (3.5) is true for $n$. Combining this with (3.6) and (3.8), we get

$$
\begin{aligned}
\|u(t)\|_{L^{r}} \leq & M e^{-\gamma^{\prime}\left(t-t_{0}\right)} \rho+M\left(\frac{M^{\prime}}{\gamma^{\prime}}\right)^{q} \rho^{p q-1} \int_{t_{0}}^{t} e^{-\gamma^{\prime}(t-s)} \sup _{[s, \infty)}\|u(\tau)\|_{L^{r}} d s \\
\leq & M e^{-\gamma^{\prime}\left(t-t_{0}\right)} \rho+2 M^{2}\left(\frac{M^{\prime}}{\gamma^{\prime}}\right)^{q} \rho^{p q} \int_{t_{0}}^{t} e^{-\gamma^{\prime}(t-s)} e^{-\gamma\left(s-t_{0}\right)} d s \\
& +M^{2} 2^{-n+1}\left(\frac{M^{\prime}}{\gamma^{\prime}}\right)^{q} \rho^{p q} \int_{t_{0}}^{t} e^{-\gamma^{\prime}(t-s)} d s \\
= & M e^{-\gamma^{\prime}\left(t-t_{0}\right)} \rho \\
& +\left[2 M\left(\frac{M^{\prime}}{\gamma^{\prime}}\right)^{q} \frac{1}{\gamma^{\prime}-\gamma} \rho^{p q-1}\right] e^{-\gamma\left(t-t_{0}\right)} \rho M \\
& -\left[2 M\left(\frac{M^{\prime}}{\gamma^{\prime}}\right)^{q} \frac{1}{\gamma^{\prime}-\gamma} \rho^{p q-1}\right] e^{-\gamma^{\prime}\left(t-t_{0}\right)} \rho M \\
& -\left[M\left(\frac{M^{\prime}}{\gamma^{\prime}}\right)^{q} \frac{1}{\gamma^{\prime}} \rho^{p q-1}\right] e^{-\gamma^{\prime}\left(t-t_{0}\right)} 2^{-n+1} \rho M+\left[M\left(\frac{M^{\prime}}{\gamma^{\prime}}\right)^{q} \frac{1}{\gamma^{\prime}} \rho^{p q-1}\right] 2^{-n+1} M \rho \\
\leq & 2 M e^{-\gamma\left(t-t_{0}\right)} \rho+2^{-n} M \rho,
\end{aligned}
$$


since $\left[5 M\left(\frac{M^{\prime}}{\gamma^{\prime}}\right)^{q} \frac{1}{\gamma^{\prime}-\gamma} \rho^{p q-1}\right]<1 / 2$, which proves (3.5). Letting $n \rightarrow \infty$ in (3.5), we obtain the estimate

$$
\|u(t)\|_{L^{r}} \leq 2 M e^{-\gamma\left(t-t_{0}\right)} \rho .
$$

Combining (3.10) with (3.8) gives

$$
\|v(s)\|_{L^{r q}}^{q} \leq 2 M\left(\frac{M^{\prime}}{\gamma^{\prime}}\right)^{q} \rho^{p q} e^{-\gamma\left(s-t_{0}\right)} .
$$

Following [3], we define the family of spaces $X_{\alpha}=D\left((-\Delta)^{\alpha}\right), \alpha \geq 0$, with $X_{\alpha} \subset L^{r}(\Omega)$. For $e^{t \Delta}$ the following estimate holds:

$$
\left\|e^{t \Delta} u\right\|_{X_{\alpha}} \leq M^{\prime \prime} t^{-\alpha} e^{-\gamma^{\prime} t}\|u\|_{L^{r}}
$$

which yields

$$
\|u(t)\|_{X_{\alpha}} \leq M e^{-\gamma\left(t-t_{0}\right)}\left\|u\left(t_{0}\right)\right\|_{L^{r}}+M^{\prime \prime} \int_{t_{0}}^{t}(t-s)^{-\alpha} e^{-\gamma^{\prime}(t-s)}\|v(s)\|_{L^{r q}}^{q} d s
$$

Combining (3.12) and (3.11) gives

$$
\|u(t)\|_{X_{\alpha}} \leq C e^{-\gamma\left(t-t_{0}\right)} \rho, \quad C>1 .
$$

A similar estimate can be obtained for $v$ :

$$
\|v(t)\|_{X_{\alpha}} \leq C^{\prime} e^{-\gamma p\left(t-t_{0}\right)} \rho^{p}, \quad C^{\prime}>1
$$

Finally, by choosing $r>n / 2,1>\alpha>n / 2 r$ we have $X_{\alpha} \hookrightarrow C^{0}$, which implies (3.1). The proof of (3.2) follows by interchanging $t$ and $-t, u$ and $v$, and $p$ and $q$.

From the proof of Lemma 3.1 it immediately follows that also

$$
\sup _{x \in \bar{\Omega}}|\nabla u(x, t)| \leq C_{1}^{\prime} e^{-\gamma\left(t-t_{0}\right)}, \quad \sup _{x \in \bar{\Omega}}|\nabla v(x, t)| \leq C_{2}^{\prime} e^{-\gamma p\left(t-t_{0}\right)}, t \geq t_{0},
$$

and

$$
\sup _{x \in \bar{\Omega}}|\nabla u(x, t)| \leq C_{3}^{\prime} e^{\gamma q\left(t+t_{0}\right)}, \quad \sup _{x \in \bar{\Omega}}|\nabla v(x, t)| \leq C_{4}^{\prime} e^{\gamma\left(t+t_{0}\right)},-t \geq t_{0},
$$

for some $t_{0}>0$ and constants $C_{1}^{\prime}, \ldots, C_{4}^{\prime}$, for any $0<\gamma<\lambda_{1}$. Indeed, if we choose $r>n$ and $1>\alpha>1 / 2+n / 2 r$, it follows that $X_{\alpha} \hookrightarrow C^{1}$, which proves the above estimates.

As a direct consequence of Lemma 3.1 and the above gradient estimates we can now integrate $(2.8)$ over the domain $\Omega \times \mathbf{R}$. This will give a global form of the modified Noether's Theorem.

Lemma 3.2. Let $u, v \in C^{2,1}(\bar{\Omega} \times \mathbf{R})$ be a classical solution of System $(H)$. Then

$$
\begin{aligned}
& -\int_{\mathbf{R}} \oint_{\partial \Omega} \frac{\partial u}{\partial n} \frac{\partial v}{\partial n}\left(x, n_{\Omega}\right) d S d t \\
& \quad=\int_{\mathbf{R}} \int_{\Omega}\left(\left(\alpha-\frac{n+2}{p+1}\right)|u|^{p+1}+\left(\beta-\frac{n+2}{q+1}\right)|v|^{q+1}\right) d x d t
\end{aligned}
$$

where $n_{\Omega}$ is the outward pointing normal on $\partial \Omega$. 
Proof of Lemma 3.2. The first step is to integrate (2.8) over $\Omega \times[-T, T], 0<T<$ $\infty$. This gives

$$
\begin{aligned}
\int_{\Omega \times[-T, T]} \operatorname{div} \mathbf{P} & =\oint_{\partial(\Omega \times[-T, T])}(\mathbf{P}, n) \\
& =\int_{[-T, T]} \int_{\Omega}\left(\left(\alpha-\frac{n+2}{p+1}\right)|u|^{p+1}+\left(\beta-\frac{n+2}{q+1}\right)|v|^{q+1}\right),
\end{aligned}
$$

where $n$ is the outward pointing normal on $\partial(\Omega \times[-T, T])$. Due to Lemma 3.1 one can take the limit as $T \rightarrow \infty$ on the right hand side of (3.14). The next step will be to evaluate the left hand side of (3.14) and take the limit as $T$ goes to $\infty$. The boundary of $\Omega \times[-T, T]$ splits in three different parts:

$$
\partial(\Omega \times[-T, T])=\partial \Omega \times[-T, T] \cup \Omega \times\{-T\} \cup \Omega \times\{T\},
$$

and we determine the left hand side of (3.14) on the three consecutive parts, using the boundary conditions (1.3). The normal $n$ on these three parts is given by $\left(\begin{array}{c}n_{\Omega} \\ 0\end{array}\right)$, $\left(\begin{array}{c}0 \\ -1\end{array}\right)$ and $\left(\begin{array}{l}0 \\ 1\end{array}\right)$ respectively. This yields

$$
\begin{aligned}
\oint_{\partial(\Omega \times[-T, T])}(\mathbf{P}, n)= & \int_{-T}^{T} \oint_{\partial \Omega}(\mathbf{P}, n)+\left(\int_{\Omega}(\mathbf{P}, n)\right)(T)+\left(\int_{\Omega}(\mathbf{P}, n)\right)(-T) \\
= & -\int_{-T}^{T} \oint_{\partial \Omega}\left[\frac{\partial u}{\partial n} \frac{\partial v}{\partial n}\left(x, n_{\Omega}\right)-2 t u_{t}\left(\nabla v \cdot n_{\Omega}\right)-2 t v_{t}\left(\nabla u \cdot n_{\Omega}\right)\right] \\
& +2 T H(u, v)(T)+2 T H(u, v)(-T) \\
& -\left(\int_{\Omega}(x \cdot \nabla u) v\right)(T)+\left(\int_{\Omega}(x \cdot \nabla u) v\right)(-T) \\
& -\alpha\left(\int_{\Omega} u v\right)(T)+\alpha\left(\int_{\Omega} u v\right)(-T) .
\end{aligned}
$$

The Hamiltonian $H(u, v)=\int_{\Omega} \mathcal{J}(u, v)$ is identically equal to zero for all $(x, t) \in$ $\bar{\Omega} \times \mathbf{R}$, and $u_{t}$ and $v_{t}$ are zero at $\partial \Omega$ for all $t \in[-T, T]$, which yields

$$
\begin{aligned}
\oint_{\partial(\Omega \times[-T, T])}(\mathbf{P}, n)= & -\int_{-T}^{T} \oint_{\partial \Omega} \frac{\partial u}{\partial n} \frac{\partial v}{\partial n}\left(x, n_{\Omega}\right)+\alpha\left(\int_{\Omega} u v\right)(-T)-\alpha\left(\int_{\Omega} u v\right)(T) \\
& +\left(\int_{\Omega}(x, \nabla u) v\right)(-T)-\left(\int_{\Omega}(x, \nabla u) v\right)(T) .
\end{aligned}
$$

We have

$$
\int_{\Omega} u v=\int_{\Omega} u(t) v(t) \leq\left|\int_{\Omega} u(t) v(t)\right| \leq C\|u(t)\|_{L^{\infty}}\|v(t)\|_{L^{\infty}},
$$

and

$$
\int_{\Omega}(x \cdot \nabla u) v \leq\left|\int_{\Omega}(x \cdot \nabla u) v\right| \leq C\|\nabla u\|_{L^{\infty}}\|u\|_{L^{\infty}} .
$$

It follows from Lemma 3.1 and the above gradient estimates $(3.1)^{\prime}$ and $(3.2)^{\prime}$ that

$$
\left|\int_{\Omega} u(t) v(t)\right| \leq \begin{cases}C e^{-(q+1) \gamma t}, & t \geq t_{0}, \\ C e^{-(p+1) \gamma t}, & -t \geq t_{0},\end{cases}
$$


and

$$
\left|\int_{\Omega}(x \cdot \nabla u(t)) v(t)\right| \leq \begin{cases}C e^{-(q+1) \gamma t}, & t \geq t_{0} \\ C e^{-(p+1) \gamma t}, & -t \geq t_{0} .\end{cases}
$$

This proves that $\left(\int_{\Omega} u v\right)( \pm T) \rightarrow 0$ and $\left(\int_{\Omega}(x \cdot \nabla u) v\right)( \pm T) \rightarrow 0$ as $T \rightarrow \infty$. Consequently the integral

$$
-\int_{-T}^{T} \oint_{\partial \Omega} \frac{\partial u}{\partial n} \frac{\partial v}{\partial n}\left(x, n_{\Omega}\right)
$$

converges as $T \rightarrow \infty$, and the limit is denoted by

$$
-\int_{\mathbf{R}} \oint_{\partial \Omega} \frac{\partial u}{\partial n} \frac{\partial v}{\partial n}\left(x, n_{\Omega}\right)
$$

Now we can take the limit as $T$ goes to $\infty$ in (3.14), which proves (3.13).

We shall now use the fundamental identity (3.13) to prove Theorem 1.2. From the Hopf Boundary Point Lemma (e.g., see [10], [11]) for the operators $\frac{\partial}{\partial t}-\Delta$ and $-\frac{\partial}{\partial t}-\Delta$ it follows, for non-negative solutions $u, v$, that both

$$
\frac{\partial u}{\partial n}<0, \quad \frac{\partial v}{\partial n}<0, \quad \forall(x, t) \in \partial \Omega \times \mathbf{R} .
$$

Due to (3.16) the left hand side of (3.13) is strictly negative. On the other hand, if $p$ and $q$ satisfy (1.6) it follows that the right hand side of (3.13) is non-negative, which is a contradiction, unless $u=v \equiv 0$ in $\Omega \times \mathbf{R}$. This concludes the proof of Theorem 1.2.

\section{REFERENCES}

1. Bahri, A. and Coron, J. M., On a nonlinear elliptic equation involving the critical Sobolev exponent: The effect of the topology of the domain, Comm. Pure Appl. Math. 41 (1988), 253-294. MR 89c:35053

2. Clément, Ph., Felmer, P. and Mitidieri, E., Solutions homoclines d'un système hamiltonien non-borné et superquadratique, C.R. Acad. Sci. Paris 320 (1995), 1481-1484. MR 96f:35072

3. Henry, D., The geometric theory of semilinear parabolic equations, Lecture Notes in Math., vol. 840, Springer-Verlag, New York, 1981. MR 83j:35084

4. Hulshof, J and van der Vorst, R.C.A.M., On the equation $\Delta u+u^{p}=0$, Course Notes Leiden/Delft 1991, 'Topics in Nonlinear Analysis'.

5. Mitidieri, E., A Rellich type identity and applictions, Comm. PDE 18 (1993), 125-151. MR 94c: 26016

6. Noether, E., Invariante Variations probleme, Nachr. König. Gesell. Wissen. Göttingen, Math.Phys. Kl. (1918), 235-257.

7. Olver, P.J., Applications of Lie Groups to Differential Equations, Springer-Verlag (GTM), New York, 1986; 2nd ed., 1993. MR 88f:58161; MR 94g:54260

8. Pohožaev, S.I., Eigenfunctions of the equations $\Delta u+\lambda f(u)=0$, Soviet Math. Dokl. 6 (1965), 1408-1411. MR 33:411

9. Pucci, P. and Serrin, J., A general variational indentity, Indiana Univ. Math. J 35 (1986), 681-703. MR 88b:35072

10. Protter, M. H. and Weinberger, H. F., Maximum Principles in Differential Equations, Prentice Hall: Ehgelwood Cliffs, NJ, 1967. MR 38:2935 
11. Smoller, J., Shock Waves and Reaction-Diffusion Equations, Grundlehren der Math. Wissensch., 2nd Ed., vol. 258, 1994. MR 95g:35002

12. van der Vorst, R.C.A.M., Variational Identities and applications to Differential Systems, Arch. Rat. Mech. Anal. 116 (1991), 375-398. MR 93d:35043

Delft University of Technology, Faculty of Technical Mathematics and Informatics, Delft, The Netherlands

Center for Dynamical Systems, Nonlinear Studies, Georgia Institute of Technology, Atlanta, Georgia 30332-0190 